\title{
PD-1 Checkpoint Inhibitor Associated Autoimmune Encephalitis
}

\author{
Stephanie Schneider ${ }^{a} \quad$ Silke Potthast ${ }^{b} \quad$ Paul Komminoth ${ }^{c}$ \\ Guido Schwegler $^{d}$ Steffen Böhm ${ }^{\text {a }}$ \\ ${ }^{a}$ Department of Internal Medicine, Oncology/Hematology, Limmattal Hospital Zurich, \\ Schlieren, Switzerland; ${ }^{b}$ Department of Radiology, Limmattal Hospital Zurich, \\ Schlieren, Switzerland; ' Department of Pathology, Triemli Hospital Zurich, \\ Zurich, Switzerland; ${ }^{d}$ Department of Neurology, Limmattal Hospital Zurich, \\ Schlieren, Switzerland
}

\section{Keywords}

Autoimmune encephalitis · PD-1 · Checkpoint inhibitor · Adverse event · Non-small cell lung cancer · Nivolumab

\begin{abstract}
Objective: To report first-hand narrative experience of autoimmune encephalitis and to briefly review currently available evidence of autoimmune encephalitis in cancer patients treated with immune checkpoint inhibitors. Setting: A case study is presented on the management of a patient who developed autoimmune encephalitis during nivolumab monotherapy occurring after 28 weeks on anti-PD-1 monotherapy (nivolumab $3 \mathrm{mg} / \mathrm{kg}$ every 2 weeks) for non-small cell lung cancer. Results: No substantial improvement was observed by antiepileptic treatment. After administration of $80 \mathrm{mg}$ methylprednisolone, neurologic symptoms disappeared within $24 \mathrm{~h}$ and the patient fully recovered. Conclusions: Immune checkpoint inhibitor treatment can lead to autoimmune encephalitis. Clinical trial data indicate a frequency of autoimmune encephalitis of $\geq 0.1$ to $<1 \%$ with a higher probability during combined or sequential anti-CTLA-4/anti-PD-1 therapy than during anti-PD-1 or anti-PD-L1 monotherapy. Further collection of evidence and translational research is warranted.
\end{abstract}




\section{Case Reports in Oncology}

\section{Introduction}

IgG4 monoclonal antibodies (mAb) nivolumab and pembrolizumab have been engineered to block the binding of PD-1 with ligands PD-L1 and PD-L2 in the tumor microenvironment. Nivolumab and pembrolizumab are currently FDA approved in first and/or second line for malignant melanoma, non-small cell lung cancer (NSCLC), and head and neck squamous cell carcinoma. Nivolumab is also approved for renal cell cancer, treatment-refractory classical Hodgkin lymphoma, and advanced urothelial carcinoma. Trials in many other solid and hematologic cancer types are ongoing and include several thousands of patients.

While efficacy in clinical trials and routine practice is encouraging, immune-related adverse events like pneumonitis, hepatitis, endocrinopathies, nephritis, and skin reactions have become a set of conditions oncologists have to familiarize with.

Neurologic adverse events, although observed, have been less in the spotlight so far. A recent survey, however, found toxicities involving the nervous system in $11.5 \%$ of melanoma patients treated with anti-PD-1 mAb [1]. These included conditions like oculomotor/abducens/facial nerve paresis, Guillain-Barré syndrome, and polyradiculitis. To date, 2 cases of autoimmune encephalitis (AIE) have been described in the literature, which occurred early during combined nivolumab and ipilimumab treatment and 1 case following sequential ipilimumab and lambrolizumab (pembrolizumab) [2,3]. None has been published with antiPD-1 monotherapy, and none in NSCLC. Notwithstanding the paucity of case studies in the literature, encephalitis was recently added to warnings and precautions in the prescribing information of all currently FDA-approved checkpoint inhibitors [4-7]. Here we describe our experience with AIE developed by a patient treated with nivolumab for NSCLC and briefly summarize currently available clinical data.

\section{Case Presentation}

A 78-year-old man with squamous cell carcinoma of the lung was treated with nivolumab ( $3 \mathrm{mg} / \mathrm{kg}$ every 2 weeks) as palliative second-line therapy following progression in the lung. After 2 months, substantial tumor regression was seen and response was confirmed after 4 months (Fig. 1). Immunohistochemical staining for PD-L1 showed approximately $20 \%$ positive tumor and approximately $1 \%$ positive immune cells (Fig. 2). Medical history included arterial hypertension, chronic renal failure, COPD, and an intracranial epidermoid tumor for which an R2 resection had been performed. The tumor rest in the left temporal lobe was showing no growth on brain MRIs since. As a consequence, the patient had occasionally experienced seizures with autonomic symptoms, partly associated with tonic convulsion of the right hand. However, when nivolumab was started, he had been seizure free for 3.5 years.

His medication included lamotrigine, bisoprolol, linsinopril, clorazepate, and baclofen.

Twelve days after the 14th application of nivolumab (28 weeks after the first dose), he presented at the emergency department with apathy and aphasia that had been progressing for $24 \mathrm{~h}$. On neurological examination, there was a Glasgow Coma Scale (GCS) score of 7 (eye opening: to pain, motor response: withdrawal from pain, verbal response: none), preexistent paresis of the left facial nerve, and no meningism. Strength appeared to be normal, but a positive Babinski sign on the left and recurrent myoclonuses of the extremities on both sides were seen. Physical examination was otherwise unremarkable and body temperature was normal. There were no signs of intracranial bleeding on computed tomography of the brain. 


\section{Case Reports in Oncology}

ECG showed sinus rhythm with unspecific ST-segment changes. Laboratory analysis revealed slightly decreased levels of hemoglobin $(13.7 \mathrm{~g} / \mathrm{dL})$, platelets $\left(122 \times 10^{9} / \mathrm{L}\right)$, and sodium (132 mEq/L = $132 \mathrm{mmol} / \mathrm{L}$, institutional normal range $135-145 \mathrm{mmol} / \mathrm{L})$ and elevated levels of creatinine $(1.3 \mathrm{mg} / \mathrm{dL}=116 \mu \mathrm{mol} / \mathrm{L}$, normal range $62-110 \mu \mathrm{mol} / \mathrm{L})$. Normal values were reported for white blood cell count, potassium, glucose, albumin-corrected serum calcium, CRP, liver enzymes, and TSH. Lamotrigine was within the therapeutic range. The patient was transferred to the ICU with suspected epileptic or prolonged postictal state. Antiepileptic treatment was initiated with levetiracetam and midazolam, and lamotrigine was continued. Baclofen was stopped. Due to differential diagnosis of infectious encephalitis, aciclovir was given, supplemented with amoxicillin and metronidazol because of suspected aspiration pneumonia on computed tomography scan. Tumor lesions were stable. On EEG there was moderate background slowing and focal delta slowing over the left temporal region with singular sharp waves in this region. Brain MRI did not reveal carcinomatous meningitis, metastases, stroke, or signs of encephalitis. Cerebrospinal fluid (CSF) showed slightly reduced level of glucosis, elevated lactate $(4.1 \mathrm{mmol} / \mathrm{L})$ and total protein $(1,027 \mathrm{mg} / \mathrm{L})$ and pleocytosis (16 lymphocytes $/ \mu \mathrm{L}$ ). On day 1-8 on ICU, the GCS score was fluctuating between 4 and 13. EEG after 5 days showed milder slowing and no sharp waves or other paroxysmal pattern. Diagnosis remained uncertain. EEG on day 9 showed less rhythmic left-hemispheric elements. Nevertheless, a persistent improvement of clinical condition was lacking after a total of 11 days. At the time, there was no reference in the literature to encephalitis following immune checkpoint inhibition. However, an immune-related adverse event of nivolumab was hypothesized and treatment with corticosteroids was initiated. Within $24 \mathrm{~h}$ after administration of methylprednisolone $(1.33 \mathrm{mg} / \mathrm{kg}$ body weight), the patient presented with GCS 15. He was mobilized and quickly returned to his baseline neurologic state. Serum antibody tests were negative (Anti-Hu, -Ri, -Yo, -amphiphysin, -CV2 (CRMP5), -Ta/Ma2, -Ma1, -SOX1, -GAD65; NMDAR not done). Corticosteroids were tapered over 9 weeks. Anti-infectious treatment was stopped when microbiological results of blood and CSF came back negative. Lamotrigine was continued and levetiracetam was tapered and discontinued. Repeat EEG 7 weeks after the first diagnosis was comparable to an EEG 2 years before. Eight months after initiation of treatment for AIE, the patient's neurological state remained at his normal level and there was no sign of tumor progression 9 months after discontinuation of nivolumab. The patient died 9 months after discontinuation of nivolumab because of bacterial pneumonia.

\section{Discussion}

We present a case of encephalitis developed after 28 weeks of anti-PD-1 monotherapy. Diagnostic criteria for probable AIE proposed by an international working group are met [8] and checkpoint inhibitor treatment is the most likely cause. Checkpoint inhibitor-associated AIE has been nonexistent in the literature until recently and the diagnosis is still a challenge to make [8]. To date, there is one paper describing two cases after a single dose each of antiPD-1 (nivolumab) and anti-CTLA-4 (ipilimumab) and one case report of a likely AIE after sequential ipilimumab and lambrolizumab (pembrolizumab) [2, 3]. The common experience of these published three cases of anti-CTLA- 4 and anti-PD-1 and the presented case of antiPD-1 monotherapy is the resolution of neurologic impairment, following immunosuppressive treatment in three of four. Interestingly, all patients had a tumor response. In contrast, the onset of this AIE was rather late (after 28 weeks), as a recent review found a median 
time of onset of neurologic adverse events of 6 weeks [9]. This comparably little evidence in the literature contrasts with the recent FDA-approved amendments of the prescribing information of anti-PD-1 mAbs nivolumab, pembrolizumab, and also the first approved antiPD-L1 mAb atezolizumab. In all of them, as well as in the prescribing information of antiCTLA-4 mAb ipilimumab, encephalitis/immune-mediated encephalitis is highlighted under warnings and precautions or adverse events. Therein, it is reported to have occurred in clinical trials with a frequency of $\geq 0.1$ to $<1 \%$ [4-7]. The risk seems to be higher with combined immune checkpoint blockade but, as described, can also happen with anti-PD-1 monotherapy.

There is a need to rapidly learn about this syndrome which includes a call for CSF and serum banking in suspected cases for analysis in research laboratories. Future guideline development should consider checkpoint inhibitor-associated AIE as a specific clinical syndrome.

By all means, it is important to collect, to report, and to build on $n=1$ experience in this young area of cancer immunotherapy.

\section{Acknowledgment}

The patient was treated with nivolumab in an early access program by Bristol-Myers Squibb.

\section{Statement of Ethics}

The patient gave informed consent to the publication of the report.

\section{Disclosure Statement}

The statements in this report reflect the opinion of the authors.

\section{References}

-1 Zimmer L, Goldinger SM, Hofmann L, Loquai C, Ugurel S, Thomas I, Schmidgen MI, Gutzmer R, Utikal JS, Goeppner D, Hassel JC, Meier F, Tietze JK, Forschner A, Weishaupt C, Leverkus M, Wahl R, Dietrich U, Garbe C, Kirchberger MC, Eigentler T, Berking C, Gesierich A, Krackhardt AM, Schadendorf D, Schuler G, Dummer R, Heinzerling LM: Neurological, respiratory, musculoskeletal, cardiac and ocular side-effects of anti-PD-1 therapy. Eur J Cancer 2016;60:210-225.

-2 Williams TJ, Benavides DR, Patrice K-A, Dalmau JO, Ribeiro de Avila AL, Le DT, Lipson EJ, Probasco JC, Mowry EM: Association of autoimmune encephalitis with combined immune checkpoint inhibitor treatment for metastatic cancer. JAMA Neurol 2016;73:928-933.

-3 Mandel JJ, Olar A, Aldape KD, Tremont-Lukats IW: Lambrolizumab induced central nervous system (CNS) toxicity. J Neurol Sci 2014;344:229-231.

$4 \quad$ Full prescribing information Opdivo ${ }^{\mathrm{TM}}$ (nivolumab).

http://www.accessdata.fda.gov/drugsatfda_docs/label/2016/125554s022lbl.pdf, Bristol-Myers Squibb Company, 2016.

$5 \quad$ Full prescribing information Keytruda ${ }^{\mathrm{TM}}$ (pembrolizumab). http://www.accessdata.fda.gov/drugsatfda_docs/label/2016/125514s012lbl.pdf, Merck Sharp \& Dohme Corp., 2016. 


\section{Case Reports in Oncology}

$6 \quad$ Full prescribing information Tecentriq ${ }^{\mathrm{TM}}$ (atezolizumab).

http://www.accessdata.fda.gov/drugsatfda_docs/label/2016/761041s000lbl.pdf, Genentech Inc., 2016.

$7 \quad$ Full prescribing information Yervoy ${ }^{\mathrm{TM}}$ (ipilimumab) http://www.accessdata.fda.gov/drugsatfda_docs/label/2015/125377s073lbl.pdf, Bristol-Myers Squibb Company, 2015.

-8 Graus F, Titulaer MJ, Balu R, Benseler S, Bien CG, Cellucci T, Cortese I, Dale RC, Gelfand JM, Geschwind M, Glaser CA, Honnorat J, Haeftberger R, lizuka T, Irani SR, Lancaster E, Leypoldt F, Pruess H, Rae-Grant A, Reindl M, Rosenfeld MR, Rostasy K, Saiz A, Venkatesan A, Vincent A, Wandinger K-P, Waters P, Dalmau J: A clinical approach to diagnosis of autoimmune encephalitis. Lancet Neurol 2016;15:391-404.

-9 Cuzzubbo A, Javeri F, Tissier M, Roumi A, Barlog C, Doridam J, Lebbe C, Belin C, Ursu R, Carpentier AF: Neurological adverse events associated with immune checkpoint inhibitors: review of the literature. Eur J Cancer 2017;73:1-8.
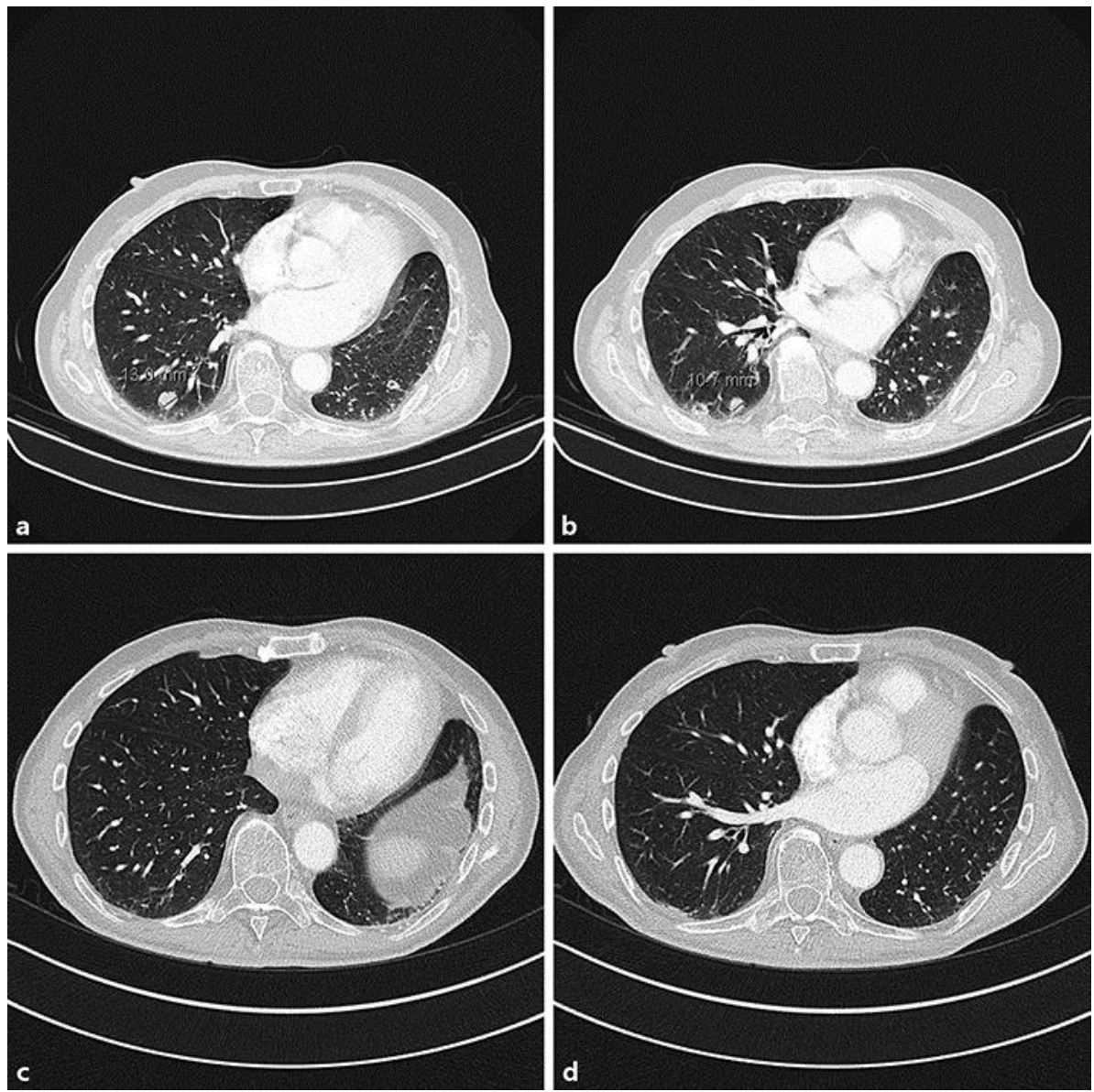

Fig. 1. Radiologic response to nivolumab: computed tomography scan shows pulmonary tumor progression after first-line palliative chemotherapy for metastatic non-small cell lung cancer in the right lower lobe (a) and in the apical segment of the right lower lobe (b). Persistent tumor regression is seen 4 months after cessation of nivolumab in the right lower lobe (c) and in the apical segment of the right lower lobe (d). 


\section{Case Reports in Oncology}

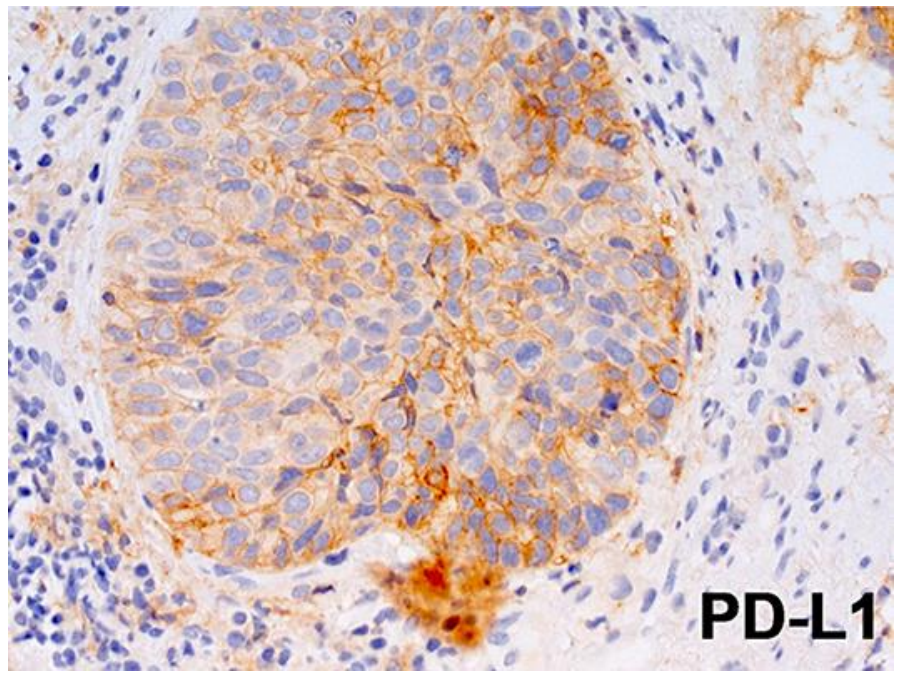

Fig. 2. PD-L1 immunohistochemical staining: representative image of PD-L1 immunohistochemical staining of squamous cell lung cancer (diagnostic biopsy). There is a patchy pattern of PD-L1 staining on approximately $20 \%$ of tumor cells with an intensity of $1-2+$. Less than $1 \%$ of the tumor is infiltrated by PD-L1positive immune cells on all slides. 